\title{
LOS NUEVOS ESCENARIOS DE TRANSMISIÓN DE LA FIEBRE HEMORRÁGICA ARGENTINA DESDE LA INTRODUCCIÓN DE LA VACUNA A VIRUS JUNÍN VIVO ATENUADO (CANDID\#1): UNA EXPERIENCIA EN TRABAJADORES GOLONDRINAS
}

\author{
NEW TRANSMISSION SCENARIOS OF THE ARGENTINE HEMORRHAGIC FEVER SINCE \\ THE INTRODUCTION OF THE LIVE ATTENUATED JUNIN VIRUS VACCINE (Candid \# 1): \\ AN EXPERIENCE IN MIGRANT WORKERS
}

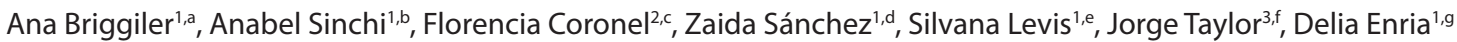

Instituto Nacional de Enfermedades Virales Humanas “Dr. Julio Maiztegui”, Pergamino, Argentina.

Programa Ampliado de Inmunizaciones. Ministerio de Salud de Santiago del Estero, Argentina.

Registro Nacional de Trabajadores y Empleadores Agrarios (RENATEA), Argentina.

Médica infectóloga, magíster en Epidemiología; ${ }^{\mathrm{b}}$ médica especialista en Medicina General; ${ }^{\mathrm{c}}$ médica especialista en Medicina Familiar; ${ }^{\mathrm{d}}$ licenciada en Enfermería; e bioquímica, doctora en Ciencias Biológicas; ${ }^{f}$ médico veterinario; ${ }^{9}$ médica infectóloga, magíster en Salud Pública.

Recibido: 14-02-15; Aprobado: 18-03-15

\begin{abstract}
RESUMEN
La fiebre hemorrágica Argentina (FHA) es una enfermedad viral aguda grave causada por el virus Junín, de la familia Arenaviridae. El área endémica de la FHA coincide geográficamente con el mayor complejo agroindustrial cerealero de exportación del Argentina. Desde la implementación de la vacunación con Candid\#1, se logró una importante reducción de la incidencia y se modificaron los patrones de riesgo. Un estudio previo permitió caracterizar estos cambios e identificar tres escenarios de transmisión: clásico, emergente-reemergente y viajero. Dentro de este último escenario se incluyen los trabajadores migrantes estacionales que se desplazan cada año, principalmente desde la provincia de Santiago del Estero, al área endémica para trabajar en el despanojado de maíz. Con el objetivo de brindar protección a este grupo de trabajadores se inició una campaña de prevención que incluyó: capacitación de personal de salud de esta provincia, educación para la salud e inmunización con vacuna Candid\#1. Se vacunaron 3021 trabajadores. Previo a la vacunación, se tomaron muestras de suero en un grupo de 104 voluntarios. Se realizó la detección de anticuerpos neutralizantes específicos para virus Junín en el total de las mismas y $6(5,76 \%)$ arrojaron resultado positivo. El inesperado hallazgo de un elevado porcentaje de trabajadores con anticuerpos, nos sugiere la necesidad de valorar varias hipótesis: a) que el resultado sea producto de un muestreo no probabilístico; b) que podría tratarse de personas que enfermaron en viajes previos, c) o que se vacunaron en viajes previos; d) considerar esta región como un escenario emergente.
\end{abstract}

Palabras clave: Virus Junin; Fiebre hemorrágica argentina; Arenavirus; Prevención \& control; Salud pública (fuente: DeCS BIREME).

\begin{abstract}
The Argentine hemorrhagic fever (AHF) is a severe acute viral disease caused by the Junin virus of the Arenaviridae family. The AHF endemic area coincides geographically with the largest grain export agro-industrial complex of the country [Argentina]. Since the implementation of vaccination with the Candid \#1 vaccine, a significant reduction in incidence was achieved and risk patterns were modified. A previous study allowed characterizing these changes and identifying three transmission scenarios: classic, emergent-reemergent, and traveler. The latter scenario includes seasonal migrant workers who move each year, mainly from the province of Santiago del Estero, the endemic area to work in the detasseling of maize. With the objective of protecting this group of workers, a prevention campaign was initiated which included: capacity building of health personnel in the province, health education, and immunization with the vaccine Candid \#1. 3,021 workers were vaccinated. Prior to vaccination, serum samples were taken from a group of 104 volunteers. Tests for neutralizing antibodies specific to the Junin virus were performed and 6 $(5.76 \%)$ tested positive. The unexpected finding of a high percentage of workers with antibodies suggests the need to evaluate several hypotheses: a) that the result is the product of non-probabilistic sampling; b) that it could be people who fell ill in previous travels, c) or who were vaccinated in previous travels; or d) consider this region as an emerging scenario.
\end{abstract}

Key words: Junin virus; Hemorrhagic fever, argentinian; Arenavirus; /Prevention \& control, Public health (source: MeSH NLM).

Citar como: Briggiler A, Sinchi A, Coronel F, Sánchez Z, Levis S, Taylor J, et al. Los nuevos escenarios de transmisión de la fiebre hemorrágica argentina desde la introducción de la vacuna a virus Junín vivo atenuado (candid\#1): una experiencia en trabajadores golondrinas. Rev Peru Med Exp Salud Publica. 2015;32(1):165-71. 


\section{INTRODUCCIÓN}

Lafiebrehemorrágicaargentina (FHA) es unaenfermedad viral grave causada por el virus Junín, perteneciente a la familia Arenaviridae. Actualmente hay más de 20 arenavirus reconocidos, de los cuales ocho se han encontrado asociados con enfermedad en humanos: en Sudamérica, el virus Junín (JUNV -fiebre hemorrágica argentina- FHA); Machupo (MACV-fiebre hemorrágica boliviana- $\mathrm{FHB}$ ); Chapare (CHAV-fiebre hemorrágica en Bolivia); Guanarito (GUAV-fiebre hemorrágica venezolana- FHV) y Sabia (fiebre hemorrágica en Brasil). En África, Lassa (fiebre de Lassa); Lujo (fiebre hemorrágica en África) y coriomeningitis linfocitaria (LCM) de distribución mundial ${ }^{(1,2)}$.

La FHA es endémica en cuatro provincias de la pampa húmeda de la Argentina: Buenos Aires, Santa Fe, Córdoba y La Pampa (Figura 1). Esta región es un área densamente poblada y coincide geográficamente con el mayor complejo agroindustrial cerealero de exportación del país. A pesar de que se pueden observar casos

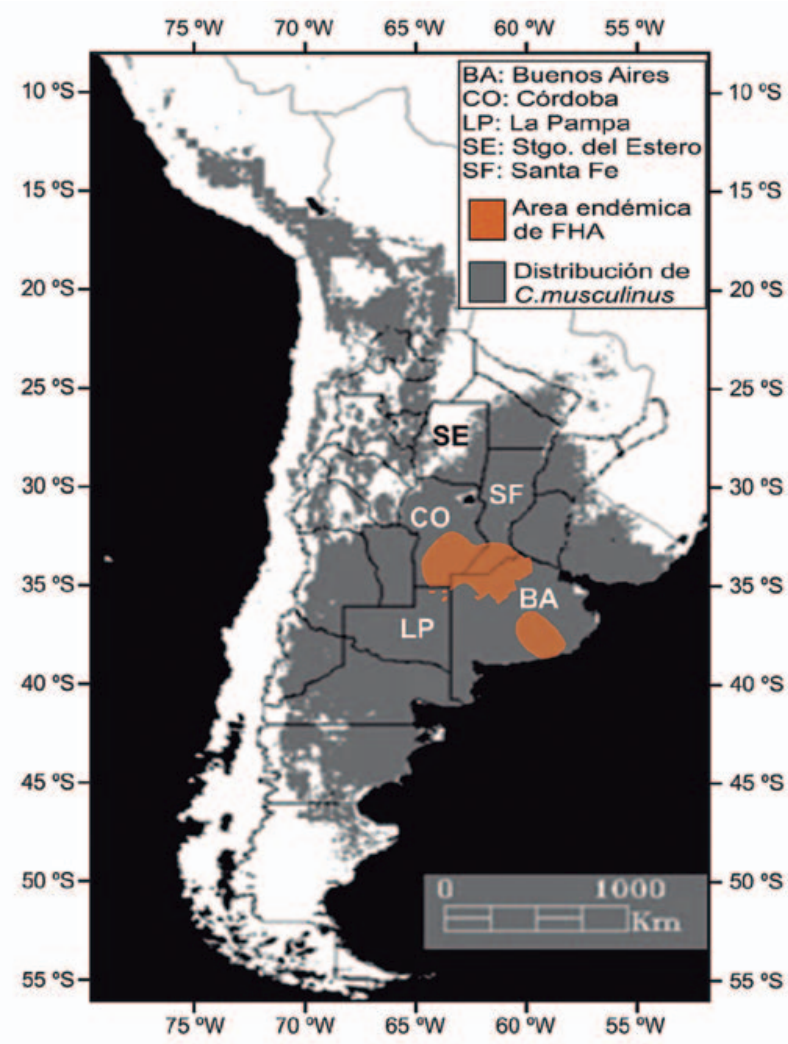

Fuente: Programa Nacional de control de la FHA y $X$. Porcasi et al

Figura 1. Área endémica de fiebre hemorrágica argentina dentro de la zona de distribución estimada de C. musculinus. Fuente: Programa Nacional de Control de la Fiebre Hemorrágica Argentina. de FHA durante todos los meses, hay anualmente brotes estacionales que se producen en el otoño y el invierno, con un pico en el mes de mayo ${ }^{(1,3)}$. Se trata de una enfermedad febril aguda, sin compromiso de las vías aéreas superiores, con escasas manifestaciones hemorrágicas durante la primera semana del inicio de los síntomas, que progresan en la segunda semana hacia síntomas hemorrágicos y/o neurológicos severos, shock y complicaciones bacterianas ${ }^{(1,3,4)}$. Puede alcanzar una letalidad de entre 16 y $30 \%$ si no es tratada con plasma inmune antes del octavo día desde el inicio de los síntomas ${ }^{(3,5,6)}$.

La infección humana resulta del contacto directo o indirecto de un individuo susceptible con roedores infectados y/o sus excretas ${ }^{(3)}$. La transmisión interhumana, si bien es infrecuente, puede ocurrir ${ }^{(3,7)}$. Es una zoonosis no erradicable debido a que su reservorio, Calomys musculinus se encuentra ampliamente distribuido en la naturaleza ${ }^{(1,3)}$. Es por ello que desde los inicios de la enfermedad muchos de los esfuerzos estuvieron dirigidos al desarrollo de una vacuna. Un proyecto de colaboración científica internacional entre los gobiernos de EE. UU. y Argentina, la Organización Panamericana de la Salud (OPS) y el Programa de Desarrollo de las Naciones Unidas (PDNU) permitió el desarrollo de la vacuna a virus Junín vivo atenuado Candid\#1. Entre 1988 y 1990 se realizó un estudio que estableció la eficacia de la vacuna en $95 \%$ ${ }^{(8)}$. Candid\#1 es una vacuna huérfana y, para asegurar su disponibilidad para la población, fue necesario lograr su producción en los laboratorios del Instituto Nacional de Enfermedades Virales Humanas (INEVH) en Argentina. Una vez lograda la producción local, un estudio puente permitió reunir los requisitos regulatorios para el registro del producto en el país ${ }^{(9)}$. Debido a la cantidad limitada de dosis de los primeros años, se inició una vacunación restringida al grupo de mayor riesgo: hombres entre 15 y 65 años que desarrollaban sus tareas en el campo $(1,8,9,10)$. A partir de 2007 Candid\#1 es incorporada al calendario nacional de inmunizaciones y desde entonces está disponible para toda la población mayor de 15 años en las cuatro provincias del área endémica.

Desde la implementación de la vacuna Candid\#1 contra la enfermedad, se logró una importante reducción de la incidencia (Figura 2). Todos los años se registran entre $25 \mathrm{y}$ 50 casos de FHA. Como es esperable, se han presentado casos en vacunados. Todos han sido formas leves de la enfermedad, que en su mayoría han evolucionado favorablemente sin tratamiento específico. Luego del inicio de la vacunación se modificaron también los patrones de riesgo. Entre los casos confirmados se viene observando un incremento en el porcentaje de mujeres, que se vacunan en menor proporción, y de niños menores de 15 años ${ }^{(10)}$. 


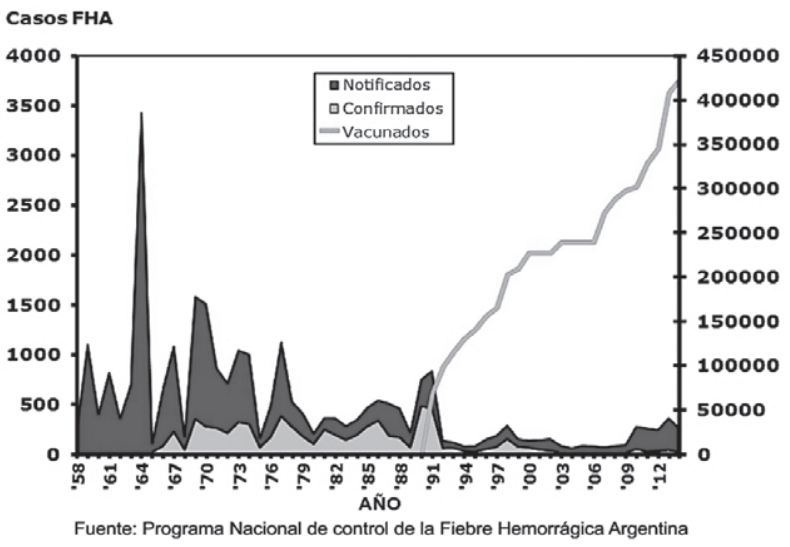

Figura 2. Distribución de casos de fiebre hemorrágica argentina (FHA), 1958-2014.

Fuente: Programa Nacional de Control de la Fiebre Hemorrágica Argentina.

También la proporción de enfermos entre personas que realizan trabajo rural disminuyó y han aumentado las cifras de casos confirmados en personas que desarrollan otras actividades económicas, no asociadas necesariamente con el campo y residentes en áreas urbanas ${ }^{(10,11)}$.

Con el principal objetivo de caracterizar estos cambios, entre los años 2011 y 2012 se realizó un estudio colaborativo multicéntrico. Como conclusión de esta investigación se definieron tres escenarios de transmisión de la FHA:

- Escenario clásico: abarca el área endémica y coincide geográficamente con el complejo agroindustrial cerealero de exportación del país (zona núcleo). El lugar de contagio y de residencia de los casos se ubican en este territorio.

- Escenario emergente-reemergente: paisajes donde acontecieron cambios en la organización social de la producción que lo hacen compatible con el escenario clásico.

- Emergente: los casos residen y se contagian fuera del área endémica pero que no viajaron en las tres semanas previas a enfermar. Áreas nuevas de transmisión.

- Reemergente: los casos residen en áreas históricas de transmisión donde no se habían notificado casos en los diez años previos.

- Escenario viajero: los casos transitaron por un escenario clásico o emergente-reemergente, pero no residen en ellos. En este escenario se unen viajeros y trabajadores que migran estacionalmente o transitan eventualmente para emplearse en tareas asociadas al complejo agroindustrial ${ }^{(12,13,14)}$.
Este último escenario, si bien no es nuevo, ha cobrado mayor notoriedad en los últimos años debido a la disminución de la incidencia en el escenario clásico. Diversos tipos de actividades ocasionan el desplazamiento anual de trabajadores desde y hacia la zona núcleo argentina. Un grupo de trabajadores que podría presentar un elevado riesgo de enfermar lo constituyen los trabajadores migrantes estacionales que se desplazan anualmente a la zona endémica para trabajar en el despanojado de maíz.

Posterior al estudio de caracterización se planteó un estudio que tuvo como objetivos: 1) Inmunizar la población migrante expuesta a riesgo de contraer FHA, 2) realizar en estudio serológico previo a la vacunación para determinar inmunidad previa y 3) Capacitar y sensibilizar al sistema de salud local para el adecuado reconocimiento de la enfermedad.

\section{ACTIVIDADES DESARROLLADAS EN EL ESTUDIO}

\section{POBLACIÓN}

Trabajadores migrantes estacionales provenientes de la provincia de Santiago del Estero que desarrollan tareas de despanojado en el área endémica de FHA. El despanojado o desflorado de maíz es un trabajo que consiste en la eliminación de las flores que producen polen, panoja, en lo alto de las plantas de maíz y arrojarlas al suelo. Es una forma de control de polinización que se emplea para hibridar o cruzar dos variedades de maíz. La mayor parte de esta actividad se realiza en forma manual, con cuadrillas de trabajadores que ingresan al sembrado y cortan las flores planta por planta (Figura 3). En Argentina, esta actividad comienza en octubre en el norte de Santiago del Estero, Catamarca y Salta, donde se

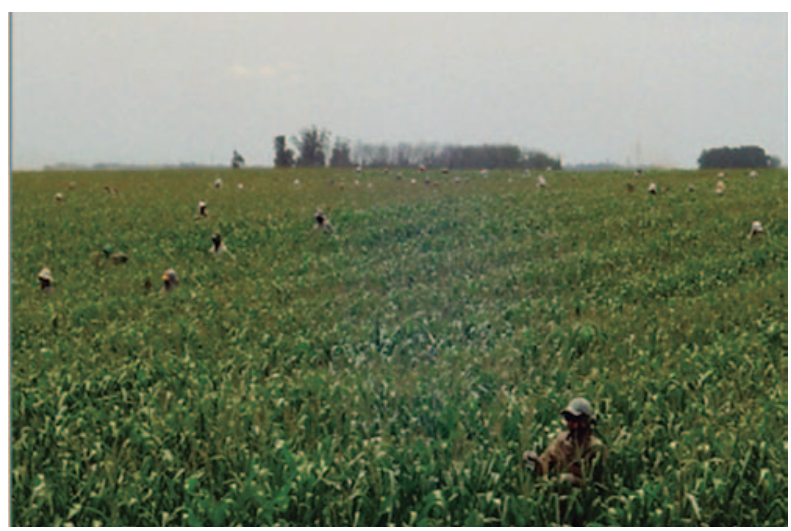

Figura 3. Despanojado manual de maíz 
extiende hasta noviembre. Desde mediados de diciembre a fines de marzo la tarea se traslada a la zona núcleo del país: norte de Buenos Aires, sur de Santa Fe y sur de Córdoba ${ }^{(15)}$. En general, los trabajadores que participan de esta actividad son residentes de otras provincias, que contratados por las empresas productoras de semillas, se desplazan todos los años durante la temporada de floración, y permanecen en la región hasta terminar la tarea. Es debido a este desplazamiento periódico que se denominan trabajadores rurales migrantes estacionales o trabajadores golondrina ${ }^{(15-17)}$. La mayor parte de esta masa laboral peregrina está constituida por residentes de la provincia de Santiago del Estero. Se estima que todos los años alrededor de 20000 personas, en su mayoría hombres, ingresan desde esa provincia para trabajar en el despanojado de maíz en la zona núcleo. Si bien no hay antecedentes de casos confirmados de FHA en trabajadores migrantes estacionales que se desempeñen en el despanojado, el riesgo de entrar en contacto con el virus de esta población no puede menospreciarse.

\section{ACTORES SOCIALES}

EI INEVH "Dr. Julio I. Maiztegui”, dependiente de la Administración Nacional de Laboratorios e Institutos de Salud -ANLIS- Ministerio de Salud Nación - es el centro coordinador del Programa Nacional de Control de la FHA. Es también laboratorio productor de la vacuna Candid\#1. Entre otras actividades, tiene como responsabilidad propiciar, colaborar y facilitar las acciones de prevención de la FHA que llevan adelante los Ministerios de Salud de las provincias. Por ello, en trabajo conjunto con el Ministerio de Salud de Santiago del Estero, personal del Programa de Médicos Comunitarios y el Registro Nacional de Trabajadores y Empleadores Agrarios (RENATEA), ente autárquico en jurisdicción del Ministerio de Trabajo, Empleo y Seguridad Social, en el marco de un Programa de Promoción de la Salud Rural, se decidió iniciar acciones de prevención de la FHA y vacunación especifica dirigidas a este grupo de trabajadores.

\section{ACTIVIDADES}

Educación para la salud y capacitación profesional.Se programó una primera instancia de capacitación en aspectos epidemiológicos, clínicos, diagnóstico, tratamiento y prevención -medidas generales y especificas (vacuna Candid\#1)- de la FHA dirigida al personal de salud local, regional y provincial. El objetivo de esta actividad fue entrenar al personal que participaría de la campaña y sensibilizar al resto del sistema de salud para la vigilancia y diagnóstico de posibles casos.
Vacunación con Candid\#1 de la población objetivo.Se organizó una primera campaña de inmunización con Candid\#1 en la localidad de Loreto, provincia de Santiago del Estero, en agosto de 2014. Esta actividad se programó en una serie de cuatro jornadas sucesivas. Se instaló el centro de actividades en el predio de la sede local de RENATEA. Para la vacunación se ubicaron puestos de información, toma de datos personales y se instaló un camión sanitario con consultorios múltiples cedido por el Ministerio de Salud. La convocatoria de los participantes la realizó personal del RENATEA en colaboración con autoridades de las intendencias locales, quienes facilitaron los medios de traslado desde el lugar de residencia de los trabajadores hasta el lugar de vacunación. Los criterios de inclusión fueron: hombres mayores de 15 años, que no hayan padecido FHA ni recibido previamente la vacuna Candid \#1 y que se desplacen anualmente a realizar actividades al área endémica de FHA. Los criterios de exclusión fueron: que no presenten condiciones médicas como cuadros febriles agudos, antecedentes de alergia a componentes de la vacuna, cuadros de inmunosupresión, haber recibido alguna vacuna o gammaglobulina en el mes previo.

Estudio serológico.- Debido a que algunos participantes podrían presentar dudas respecto a si enfermaron o si se vacunaron con Candid\#1 en viajes previos al área endémica, en el mismo lugar se ubicó un puesto de extracción de muestras de sangre para ofrecer a los trabajadores la posibilidad de chequear su estado serológico frente a virus Junín. Se utilizó un tubo al vacío, de $10 \mathrm{~mL}$, para la obtención de sangre en cada participante. La toma de la muestra se realizó en forma libre y voluntaria a aquellas personas que manifestaron su interés. Luego de explicar el objetivo del estudio, cómo se realiza y los efectos colaterales que puede tener la extracción (dolor y/o hematoma en el sitio de punción), cada participante tuvo oportunidad de aclarar dudas y luego firmar voluntariamente un consentimiento escrito. También, previo a la toma de la muestra, se interrogó a cada voluntario respecto de antecedentes de viaje a la zona endémica, de vacunación con Candid\#1 y si recordaba haber enfermado durante o inmediatamente después de alguno de sus viajes.

Las muestras de suero fueron procesadas en el laboratorio de INEVH. Se realizó la detección de anticuerpos neutralizantes específicos (ACNT) en suero. El título de ACNT se estableció como la mayor dilución del suero que inhibe el $80 \%$ de unidades formadoras de placas (ufp) de JUNV en células Vero. 


\section{RESULTADOS}

La instancia de capacitación estuvo a cargo de profesionales del INEVH. El Ministerio de Salud provincial convocó a autoridades sanitarias locales, médicos hospitalarios, médicos comunitarios, enfermeras, agentes sanitarios y vacunadores para que participasen de esta actividad. Se realizó previo al inicio de la campaña de vacunación en el hospital de Loreto. Se llevó adelante en modalidad taller, con una parte instructiva teórica. A continuación se realizó una práctica de vacunación con Candid\#1, según normas. En las jornadas posteriores se continuó con asesoría en terreno.

Durante esta primera campaña se vacunaron 3021 trabajadores provenientes de diferentes localidades del centro y sur de la provincia (Figura 4).También se les brindó información sobre la enfermedad y medidas de prevención. Previo a la inmunización, el personal de salud realizó la anamnesis clínica correspondiente y explicó posibles efectos adversos de la vacuna a cada participante.

Se obtuvieron muestras de suero de 104 personas previo a la inmunización. Se procesó el total de las muestras obtenidas por técnica de neutralización. Seis de las 104 resultaron positivas $(5,76 \%)$. Los títulos de ACNT virus Junín obtenidos, expresado como inversa de la dilución, fueron $10(n=1), 80(n=1), 160(n=3)$, y $640(n=1)$.

El $100 \%$ de los voluntarios tenían antecedentes de viaje a la zona endémica para trabajo en el despanojado de maíz. Ninguno recordaba haber padecido FHA. Ninguno reportó haber padecido alguna enfermedad febril durante su permanencia en la zona endémica ni inmediatamente después de su retorno. Todos negaron haber recibido vacuna Candid\#1 con anterioridad.

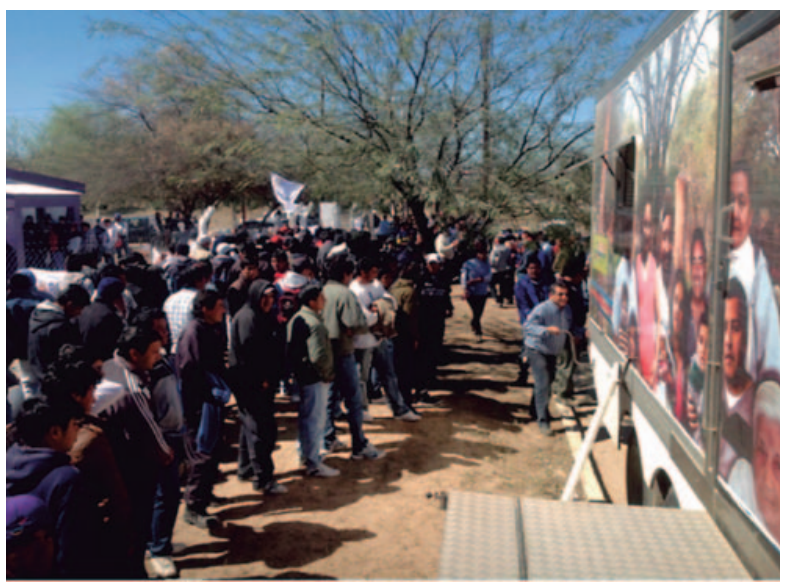

Figura 4. Vacunación en Loreto (agosto 2014)

\section{DISCUSIÓN}

No hay antecedentes de casos notificados de FHA que se hayan confirmado etiológicamente en trabajadores migrantes estacionales que se desempeñen en el despanojado. Sin embargo, la zona núcleo coincide también con el escenario clásico de transmisión de la FHA. En sus inicios, la enfermedad afectó de manera importante a trabajadores rurales que participaban en la cosecha manual del maíz, muchos de ellos también migrantes ${ }^{(18)}$. Por esta razón, llama la atención que no haya casos confirmados entre los trabajadores del desflore. Este hecho podría explicarse considerando que estas actividades se dan en diferentes épocas del año. La cosecha manual se realizaba en los meses de otoño, mientras que las actividades de despanojado se llevan adelante en los meses de verano, de diciembre a marzo. La época estival es una temporada con bajas densidades poblacionales de Calomys ${ }^{(1)}$, lo que disminuiría el riesgo de exposición.

Sin embargo, resulta elevado el porcentaje de trabajadores migrantes que presentaron anticuerpos contra el virus Junín. Encuestas serológicas realizadas en población rural del área endémica mostraron que la prevalencia de infección subclínica varía entre 1 y el $4,44 \%{ }^{(7,19)}$. Este hallazgo nos obliga a evaluar varias hipótesis:

1) Que el resultado sea producto de un muestreo no probabilístico y que en un estudio planificado no se repita.

2) Podría tratarse de personas inmunizadas previamente con Candid\#1 y que no lo recuerden. La vacunación debería haberse llevado a cabo en algún viaje previo a la zona endémica, ya que antes nunca estuvo disponible la vacuna en la provincia de Santiago del Estero. No es aún posible determinar por laboratorio si los anticuerpos son vacunales o debidos a infección natural. Sin embargo, llaman la atención los títulos obtenidos ya que la media geométrica observada entre los vacunados en una cohorte de seguimiento de 25 años es de 70 .

3) Otra hipótesis es que algunos trabajadores podrían haber desarrollado infección subclínica o haberse marchado delárea endémica en período de incubación y enfermado en su lugar de origen. La enfermedad pudo no haber sido adecuadamente reconocida por falta de sensibilización del sistema de salud. Esta probabilidad tiene un riesgo adicional asociado: la FHA puede ser contagiada entre personas si media contacto íntimo o por transfusión ${ }^{(7)}$. Esta situación aumenta considerablemente el riesgo de morir por FHA. La enfermedad oportunamente tratada con 
el plasma inmune tiene una letalidad menor del $1 \%$. Si no se trata correctamente la letalidad puede ascender hasta $30 \%$.

4) Una cuarta hipótesis plantea que podríamos estar frente a otro tipo de escenario. Existen publicaciones que indican que la distribución de Calomys musculinus se extiende desde el centro oeste de Bolivia hasta la Patagonia Argentina ${ }^{(20,21,22)}$. Desde la emergencia de la FHA en la década del 50, la región endémica se ha expandido geográficamente y los estudios en roedores han sugerido la posibilidad de una extensión hacia el norte ${ }^{(20)}$. Por esta razón deberíamos considerar la idea de que en realidad estemos ante un nuevo escenario emergente.

Estos datos requerirían una serie de nuevas investigaciones. Se debería intentar recuperar datos de antecedentes en estos casos positivos mediante otras metodologías de investigación tales como entrevistas en profundidad o semiestructuradas y revisión de fuentes secundarias, como archivos de vacunatorios de las demás provincias. Esta tarea es difícil porque a pesar de que la vacunación se registra en forma nominal, la información, en general, no está digitalizada.

Para evaluar la tercera hipótesis habría que realizar una búsqueda retrospectiva de casos en historias clínicas con cuadros compatibles y con el antecedente de viaje. Pero estamos hablando de decenas de miles de personas por año que desarrollan estos desplazamientos temporales, lo que hace dificultoso determinar por dónde iniciar la búsqueda y durante qué período realizarla.

Respecto de la última hipótesis, sería importante realizar una encuesta serológica en la población de Santiago del Estero, incluida la no migrante, mujeres y niños. En caso de encontrar personas seropositivas sin antecedentes de viajes, debería también implementarse capturas de roedores para intentar buscar actividad de virus Junín en la región y confirmar endemicidad. Estudios previos en Calomys musculinus capturados en Santiago del Estero no mostraron evidencias de actividad del virus Junín.

Finalmente, como logro de este trabajo, para diciembre de 2014 ya se habían vacunado más de 9000 trabajadores. Consideramos que si bien todavía no se ha alcanzado la meta de $100 \%$ de cobertura, la medida ha sido altamente exitosa, ya que no solo se brindó protección mediante la información sobre la enfermedad y la inmunización de la población expuesta, sino que además se generó estado de alerta y capacitación del sistema de salud local.

Este artículo expone un ejemplo de cómo el resultado de una investigación en salud pública resultó en medidas concretas destinadas al control de una fiebre hemorrágica viral. Del mismo modo, el cumplimiento de las metas propuestas solo fue posible gracias al trabajo intersectorial coordinado. Se iniciaron acciones de prevención de la FHA en un nuevo escenario, se plantearon hipótesis que sugieren nuevas líneas de investigación y se sumaron nuevos actores sociales al Programa Nacional de Control de la FHA.

Contribuciones de autoría: D. Enria, A. Briggiler y A. Sinchi han participado de la concepción y diseño del artículo. A. Briggiler, A Sinchi, y Z. Sanchez participaron en el trabajo en terreno (capacitación de trabajadores de salud, recolección de datos, obtención de muestras de sangre, inmunización de la población). F Coronel y J Taylor participaron en el reclutamiento de los participantes y coordinación del equipo de salud provincial. S. Levis participó en la obtención de resultados de laboratorio. El análisis e interpretación de datos los realizaron D. Enria, A. Briggiler y A. Sinchi. La redacción del artículo estuvo a cargo de A. Briggiler y A. Sinchi. La revisión crítica la realizó $D$. Enria. La aprobación de su versión final estuvo a cargo de D. Enria y A.Briggiler.

Fuentes de financiamiento: autofinanciado.

Conflictos de interés: los autores declaran no tener conflictos de interés.

\section{REFERENCIAS BIBLIOGRÁFICAS}

1. Castilla V, Enria D. Arenaviridae. En: Basualdo JA, Coto CE y de Torres RA. Microbiología Médica. 3ra. ed. Buenos Aires: Atlante; 2013.

2. Blumberg L, Enria D, Bausch DG. Viral Haemorrhagic Fevers. En: Farrar J, Hotez PJ, Junghanss T, Kang G, Lalloo D, White NJ. Manson's Tropical Diseases. 23ava ed. 2014. p. 171-94.

3. Enria D, Briggiler AM, Feuillade MR. An overview of the epidemiological, and preventive hallmarks of Argentine haemorrhagic fever (Junin virus). Bull Inst Pasteur.1998;96:103-14.

4. Sabattini MSy MaizteguiJI. Adelantos en medicina: fiebre hemorrágica argentina. Medicina (B. Aires).1970;30(1):111-28.

5. Maiztegui JI, Fernandez NJ, de Damilano AJ. Efficacy of immune plasma in treatment of Argentine haemorrhagic fever and association between treatment and a late neurological syndrome. Lancet. 1979 Dec 8;2(8154):1216-7.
6. Enria DA, Briggiler AM, Fernandez NJ, Levis SC, Maiztegui JI. Importance of dose of neutralising antibodies in treatment of Argentine haemorrhagic fever with immune plasma. Lancet. 1984 Aug 4;2(8397):255-6

7. Briggiler A, Enria D, Feuillade MR, Maiztegui J. Contagio interhumano e infección inaparente por virus Junín en matrimonios del área endémica de fiebre hemorrágica argentina (FHA). Medicina (B. Aires). 1987;47(6):565. 
8. Maiztegui JI, McKee KT Jr, Barrera Oro JG, Harrison LH, Gibbs PH, Feuillade MR, et al. Protective efficacy of a live attenuated vaccine against Argentine hemorrhagic fever. AHF Study Group. J Infect Dis. 1998 Feb;177(2):277-83.

9. Enria DA, Ambrosio AM, Briggiler AM, Feuillade MR, Crivelli E; Study Group on Argentine Hemorrhagic Fever Vaccine. [Candid\#1 vaccine against Argentine hemorrhagic fever produced in Argentina. Immunogenicity and safety]. Medicina (B Aires). 2010;70(3):215-22. [Artículo en Español]

10. Enria DA, Feuillade MR, Levis S, Briggiler AM, Ambrosio AM, Saavedra MC, et al. Impact of the vaccination of high risk population for AHF with a live attenuated Junin virus vaccine. En: Saluzzo JF, Dodet B, ed. Factors in the Emergence and Control of RodentBorne Viral Diseases (Hantaviral and Arenaviral Diseases). París: Elsevier; 1999:273-80.

11. Sinchi A, Feuillade M, Briggiler A, Calderón G, Digilio C, Mastrángelo A, et al. Cambio en los patrones de riesgo de la fiebre hemorrágica argentina desde la introducción de la vacunación. En: Libro de resúmenes: XII Congreso Argentino de la Sociedad Argentina de Infectología. Córdoba; Sociedad Argentina de Infectología; 2012. p. 110.

12. Enria DA, Mastrángelo AV, Tagliabue P, Digilio C, Sinchi A, Berro L, et al. Nuevos escenarios de transmisión de la fiebre hemorrágica argentina en las provincias de Buenos Aires y Santa Fe en la era posvacunación 2001-2010. En: Comisión Nacional
Salud Investiga. Anuario Becas de Investigación "Ramón Carrillo Arturo Oñativia” 2011. Buenos Aires; MINSA; 2013:234.

13. Mastrangelo A, Tagliabue P, Sinchi A, Feuillade M, Briggiler A, Digilio C, et al. Nuevos escenarios de transmisión en el contexto epidemiológico de la fiebre hemorrágica argentina. En: Libro de resúmenes: XII Congreso Argentino de la Sociedad Argentina de Infectología. Córdoba; Sociedad Argentina de Infectología; 2012:120.

14. Mastrangelo A, Tagliabue P, Berro L, De Carolis D, Sinchi A, Digilio C,et al. Estudio cualicuantitativo de las variables sociales que definen escenarios de transmisión de la fiebre hemorrágica argentina en las provincias de Buenos Aires y Santa Fe, 2001-2010. Salud Colect. 2010;10(2):171-84.

15. Desalvo, A. Migraciones estacionales: el caso de los trabajadores santiagueños en el despanojado de maíz (2009-2012). Trabajo y Sociedad. 2014;22:37-66.

16. Tasso A, Zurita C. Aves de paso: los trabajadores estacionales de Santiago del Estero. Trabajo y Sociedad. 2013; 21:33-47.

17. Andreani H. Migración, maíz y silencio. Aproximaciones al bilingüismo (quichua-español) de los trabajadores 'golondrina' de Santiago del Estero (Argentina). Gazeta de Antropología [Internet]. 2013 [citado el 14 de febrero del 2015];29(1). Disponible en: http://www.gazeta-antropologia. es/?p= 4167

18. Agnese G. La "peste” de los peones. Fiebre hemorrágica argentina: población e imaginario 1943-1962. En: Drovetta R, Rodríguez M. Padecimientos en grupos vulnerables del interior de Argentina: procesos históricos y actuales de salud, enfermedad y atención. Córdoba: Universidad Nacional de Córdoba; 2010. p. 75-105.

19. Weissenbacher MC, Sabattini MS, Avila MM, Sangiorgio PM, de Sensi MR, Contigiani MS, et al. Junin virus activity in two rural populations of the Argentine hemorrhagic fever (AHF) endemic area. J Med Virol. 1983;12(4):273-80.

20. Mills JN, Ellis BA, McKee KT Jr, Ksiazek TG, Oro JG, Maiztegui JI, et al. Junin virus activity in rodents from endemic and nonendemic loci in central Argentina. Am J Trop Med Hyg. 1991;44(6):589-97.

21. Porcasi X, Calderón GE, Lamfri M, Scavuzzo M, Sabattini MS, Polop JJ. Predictive distribution maps of rodent reservoir species of zoonoses in Southern America. Mastozoología Neotropical. 2005;12(2):199-216.

22. Jayat JP, Ortiz PE, Teta P, Pardiñas UF, D’Elía G. Nuevas localidades argentinas para algunos roedores sigmodontinos (Rodentia: Cricetidae). Mastozoología Neotropical. 2006;13(1):51-67.

Correspondencia: Ana Briggiler Dirección: Monteagudo 2510-Pergamino, Buenos Aires, Argentina

Teléfono: (+54) 2477433044

Correoelectrónico:abriggiler@hotmail.com 